\section{Prävention durch pränatale Allergenexposition}

\author{
Eine Allergenexposition werdender Mütter kann den Nachwuchs \\ vor Allergien schützen - diese Aussage stimmt offenbar zumindest \\ im Mausmodell.
}

O

$\mathrm{b}$ eine pränatale Allergenexposition zur primären Prävention atopischer Erkrankungen eingesetzt werden kann, ist bislang nicht bekannt. Dieser Frage ging eine Berliner Arbeitsgruppe um Dr. Kerstin Gerhold unter Leitung von Prof. Dr. Eckard Hamelmann im Mausmodell nach.

Trächtige Muttertiere wurden von Tag sieben der Tragzeit an bis zur Geburt gegenüber Ovalbumin (OVA) als Modellallergen über die Atemwege exponiert. Kontrolltiere waren gegenüber „phosphate buffered saline“(PBS) exponiert. Die Nachkommen wurden systemisch mit OVA sensibilisiert, eine Kontrollgruppe erhielt systemisch PBS. Am
Tag 52 postnatal wurde dann überprüft, $o b$ es bei den Jungtieren zu einer Sensibilisierung oder zur Toleranzinduktion gegenüber dem Allergen gekommen war.

Die Nachkommen der OVA-exponierten Muttertiere wiesen im Vergleich zu denjenigen der Kontrollmuttertiere signifikant niedrigere Serumtiter an allergenspezifischem IgE und eine Suppression von TH2-Zytokinen auf. In der broncheoalveolären Lavage waren Leukozyten und eosinophile Granulozyten deutlich erniedrigt, die Atemwegsreaktivität war nicht erhöht. Die pränatale OVA-Exposition über die Atemwege verhinderte also Sensibilisierung, Atem- wegsentzündung und Atemwegshyperrekativität bei den Jungtieren, so Gerhold. Die präventive Wirkung war langanhaltend und konnte auch an Tag 115 postnatal noch nachgewiesen werden, war also nicht allein auf mütterliche IgGAntikörper zurückzuführen.

In vitro ließ sich zeigen, dass die pränatale OVA-Exposition mit einer Suppression der TH2-Zytokine, einer Induktion des TH1-Zytokins IFN- $\gamma$, einer verminderten allergenspezifischen T-Zell-Proliferation sowie einer erhöhten Frequenz regulatorischer T-Zellen bei den Jungtieren assoziiert war. Über den Mechanismus der allergenspezifischen Toleranzinduktion könnte der protektive Effekt einer pränatalen Allergenexposition erklärt werden. $\quad a b d$

\section{Gerhold K, Avagyan A, Seib C, Wahn U,} Hamelmann E. Pränatale Allergenexposition zur Prävention einer späteren Allergen-induzierten Sensibilisierung und Atemwegsentzündung in der jungen Maus. 20. Mainzer Allergie-Workshop, Mainz, 7./8. März 2008 